\title{
Supporting Information: Efficient Modeling Workflow for Accurate Electronic Structures of Hybrid Perovskites
}

\author{
Julian Gebhardt ${ }^{1,2},{ }^{*}$ Wei $\mathrm{Wei}^{2}$, and Christian Elsässer ${ }^{1,2,3}$ \\ ${ }^{1}$ Fraunhofer IWM, Wöhlerstraße 11, 79108 Freiburg, Germany \\ ${ }^{2}$ Cluster of Excellence livMatS at FIT - Freiburg Center for Interactive \\ Materials and Bioinspired Technologies, Albert-Ludwigs-University Freiburg, \\ Georges-Köhler-Allee 105, 79110 Freiburg, Germany and \\ ${ }^{3}$ Freiburg Materials Research Center (FMF), Albert-Ludwigs-University Freiburg, \\ Stefan-Meier-Straße 21, 79104 Freiburg, Germany
}

(Dated: August 12, 2021) 


\section{INFLUENCE OF FA ORIENTATION ON STABILITY AND BAND GAP}

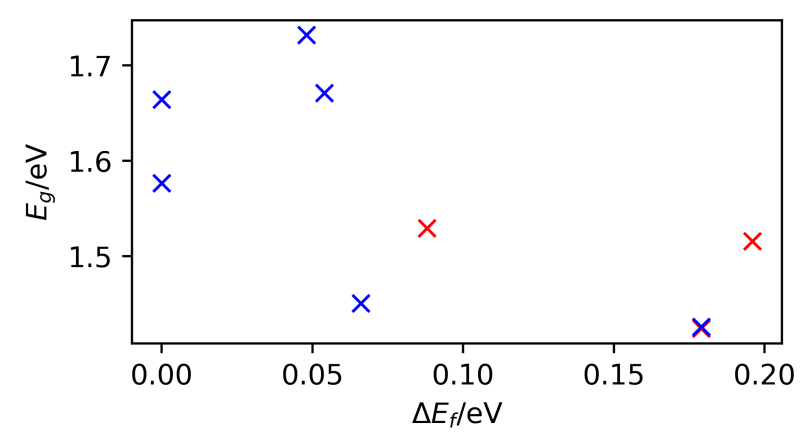

FIG. S1: Band gap $E_{g}$ versus difference in formation energy $\Delta E_{f}$ for the investigated parallel (red) and lower symmetry structures (blue).

[100]
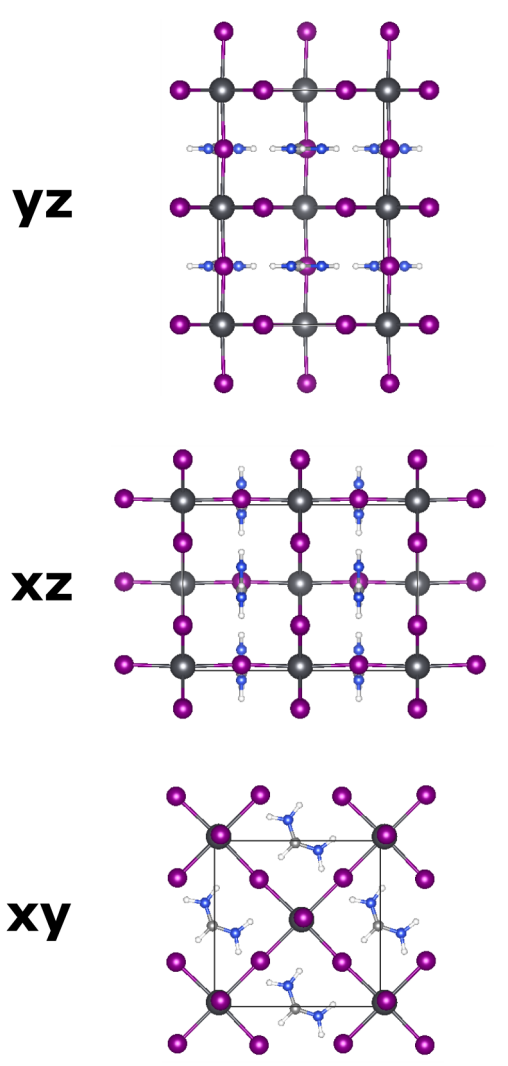

[110]
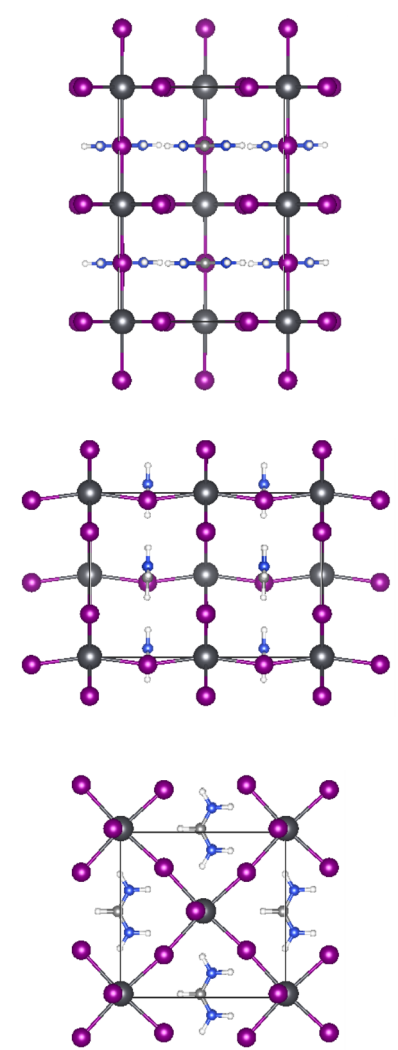

[111]
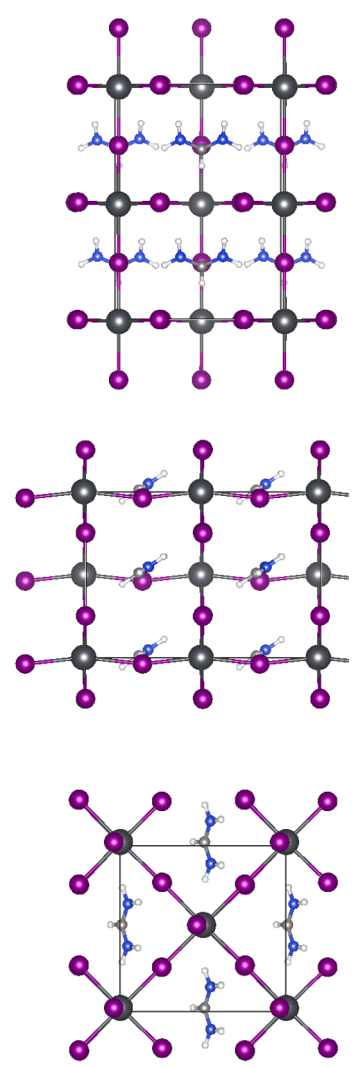

FIG. S2: Geometries of parallel $\mathrm{FAPbI}_{3}$ structures, with all FA molecules oriented along [100], [110], and [111] directions, respectively. 


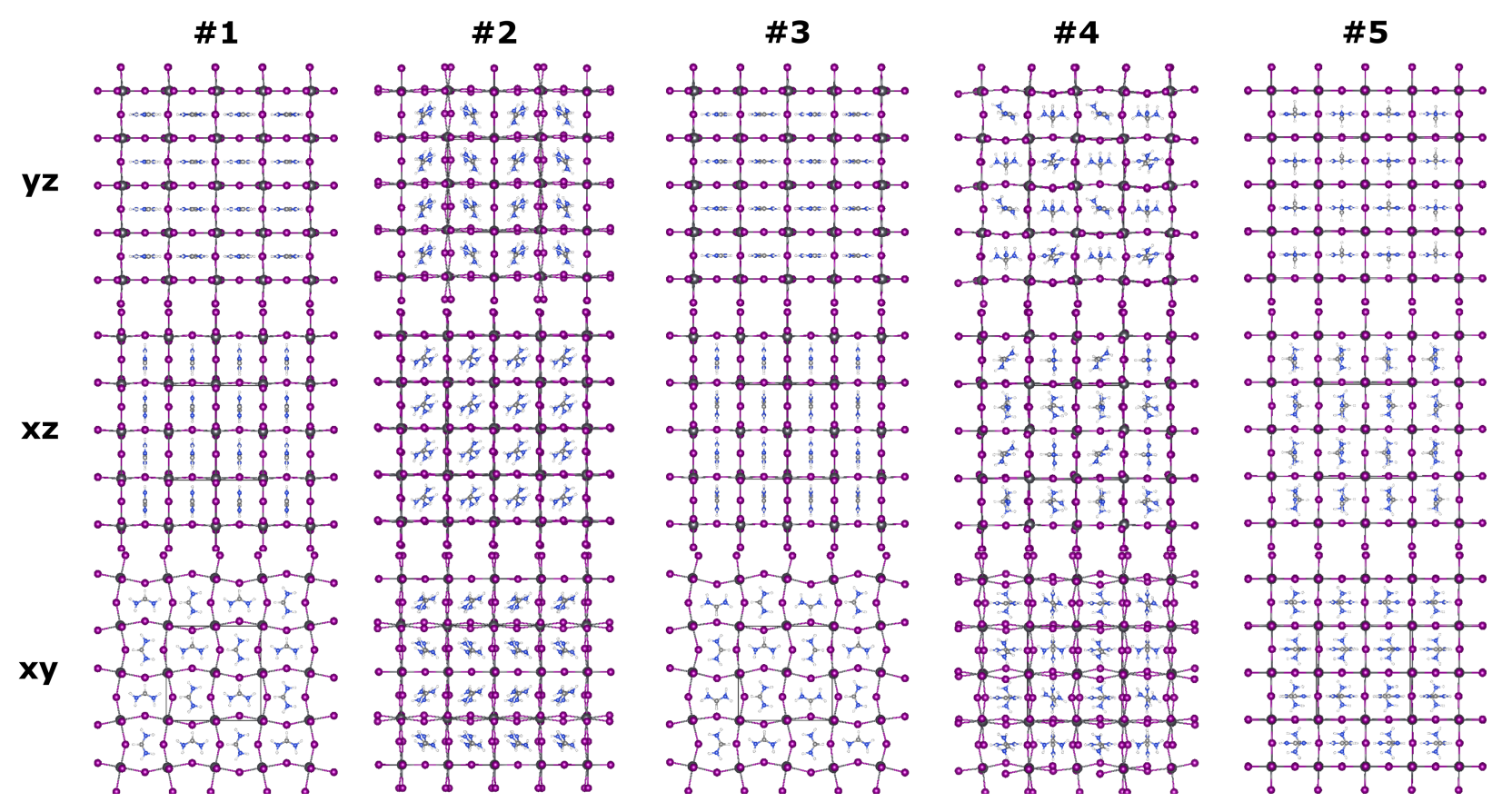

FIG. S3: Geometries of non-parallel $\mathrm{FAPbI}_{3}$ structures, leading to tilted $\mathrm{PbI}_{3}$-backbone structures. 


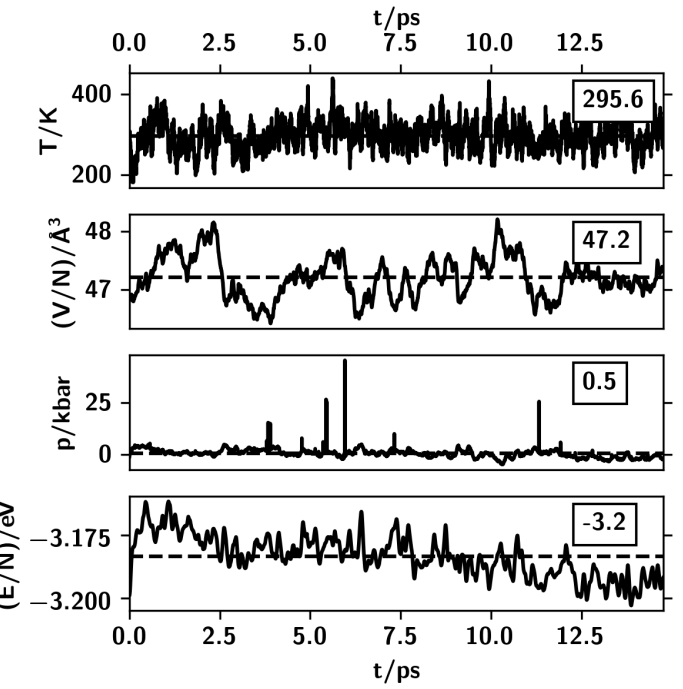

FIG. S4: Temporal evolution of temperature (T), Volume (V) per particle (N), pressure (p), and energy $(E)$ per particle in the MD-run for $\mathrm{CsPbI}_{3}$.

INFLUENCE OF TEMPERATURE ON MD
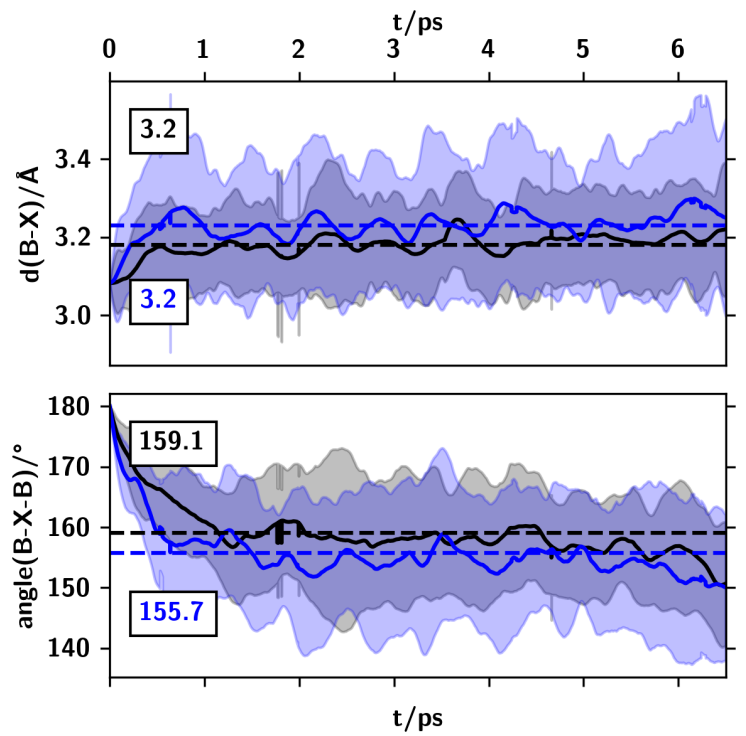

FIG. S5: Temporal evolution of bond distance and bond angle in 6.5 ps AIMD runs for $\mathrm{CsPbI}_{3}$ at $300 \mathrm{~K}$ (black) and $600 \mathrm{~K}$ (blue). 

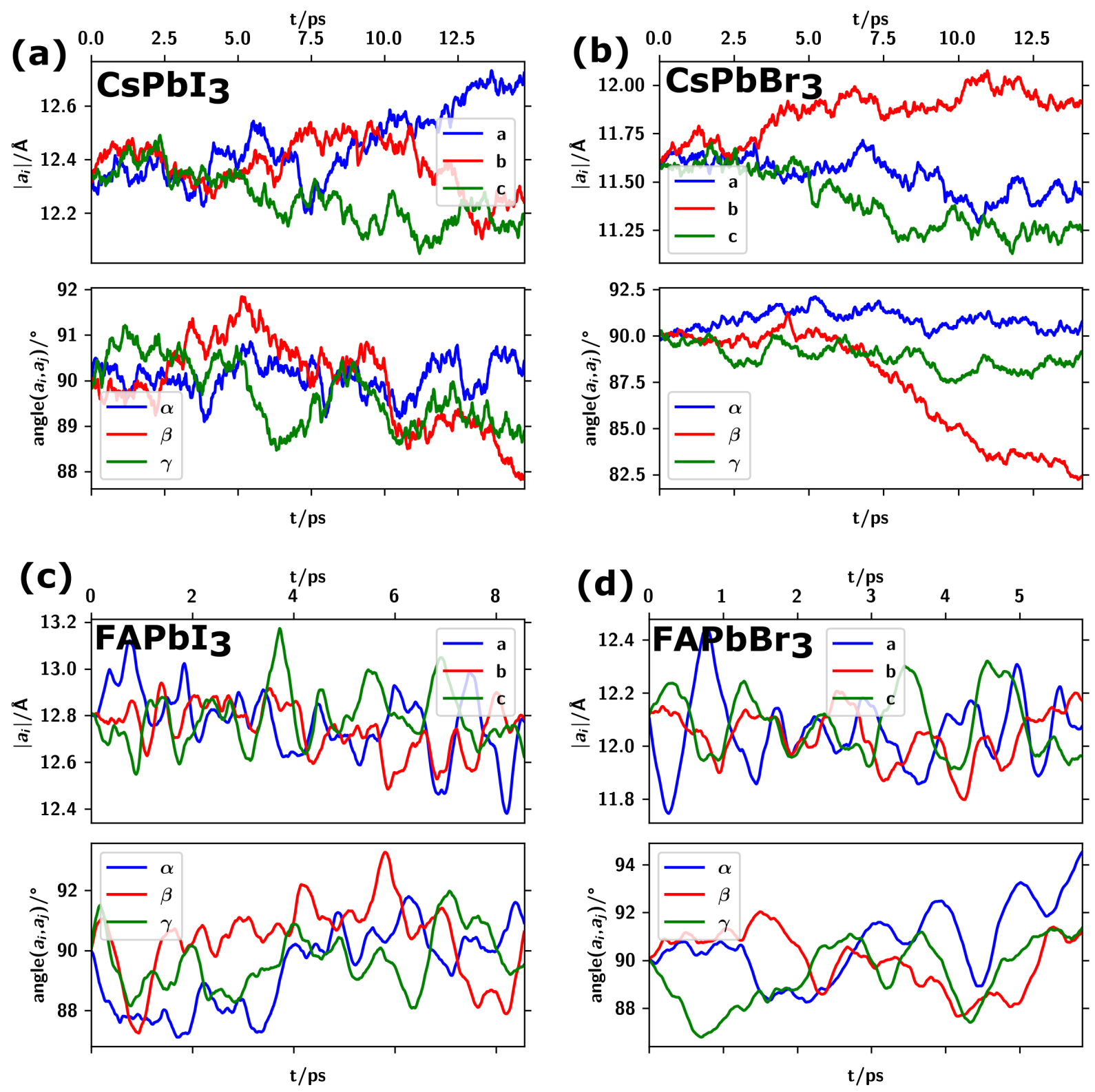

FIG. S6: Temporal evolution of lattice parameters in the AIMD runs for $\mathrm{CsPbI}_{3}, \mathrm{CsPbBr}_{3}$, $\mathrm{FAPbI}_{3}$, and $\mathrm{FAPbBr}_{3}$. 
DETAILS OF CSPBBR 3 MD-RUN
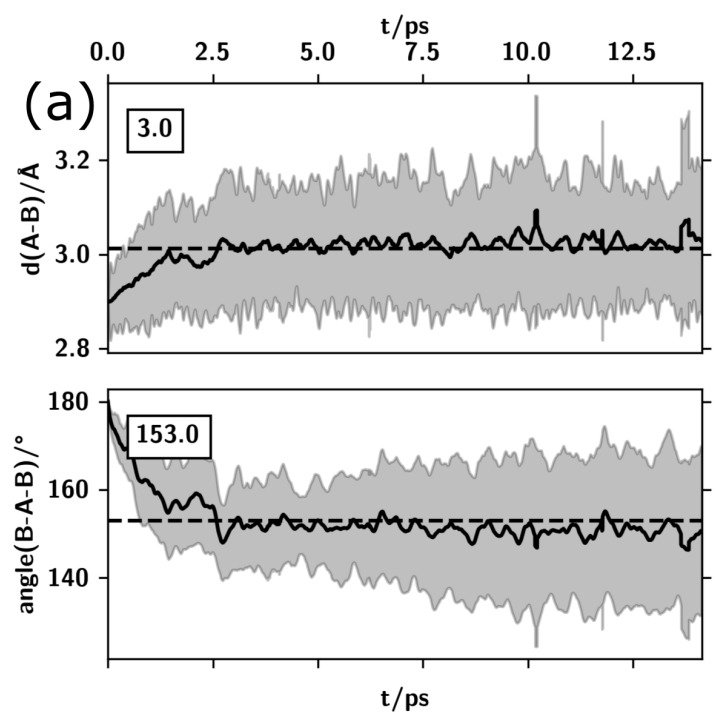

(b)

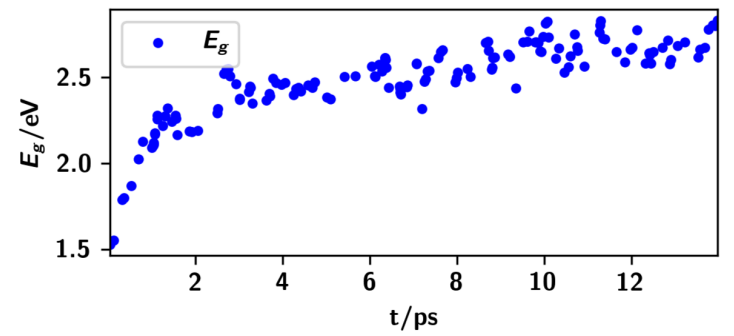

FIG. S7: (a) Pb-Br bond distance and $\mathrm{Pb}-\mathrm{Br}-\mathrm{Pb}$ bond angle. The mean value is plotted at each time step in black, the gray region indicates the standard deviation. (b) Band gap computed for static structures taken as snapshots during the AIMD run.

DETAILS OF FAPB $X_{3}$ MD-RUN
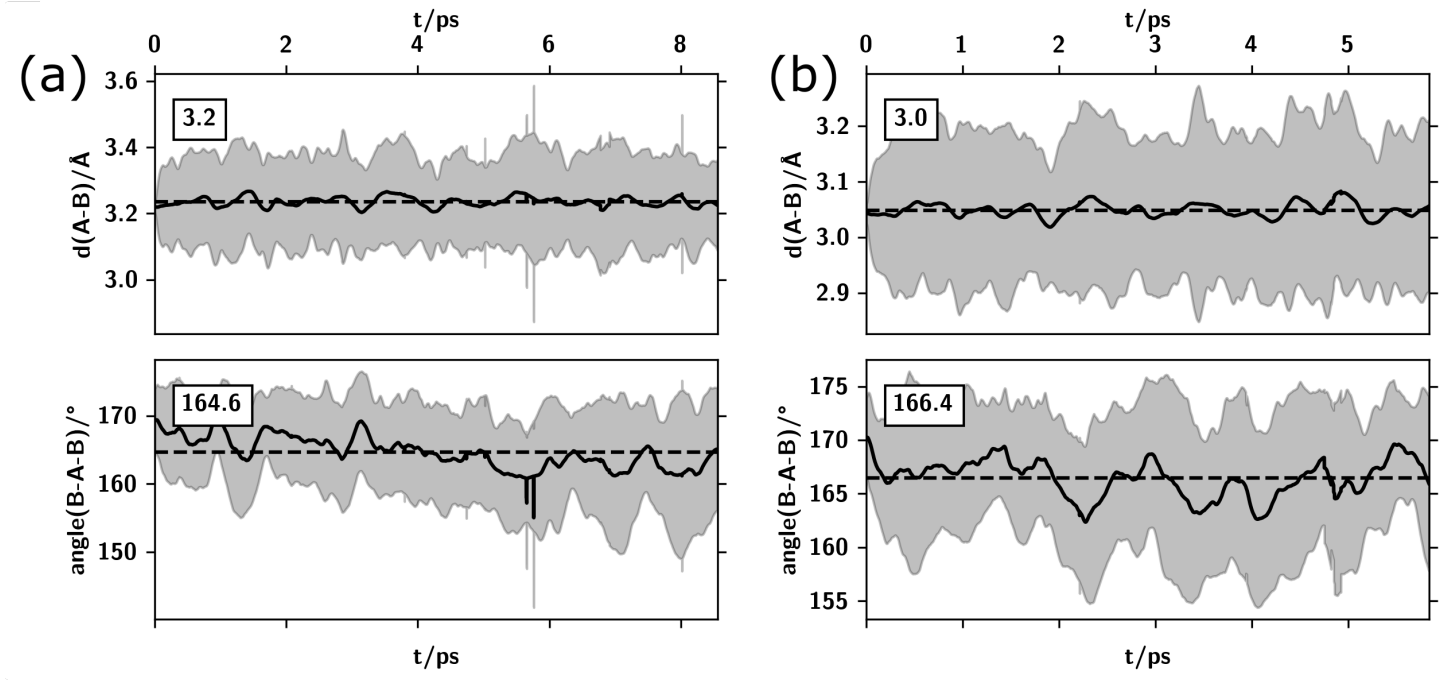

FIG. S8: $\mathrm{Pb}-X$ bond distance and $\mathrm{Pb}-X-\mathrm{Pb}$ bond angle for $\mathrm{FAPbI}_{3}$ (a) and $\mathrm{FAPbIBr}_{3}(\mathrm{~b})$. 


\section{COMPUTATIONAL DETAILS}

Static first-principles calculations of atomistic and electronic structures are performed for inorganic and hybrid perovskite crystals in the cubic $\alpha$-phase, represented by a $(\sqrt{2} \times \sqrt{2} \times 2)$ unit cell in order to enable possible octahedral tilts. The calculations are carried out with the Vienna ab initio simulation package (VASP)[1] employing projector-augmented waves[2] potentials, the semi-local Perdew-Burke-Ernzerhof (PBE)[3] exchange-correlation functional, and the TS correction[4] for dispersion interactions. An energy cutoff of $520 \mathrm{eV}$ was used for the plane-waves basis. Total-energy differences and forces on atoms for all structural

degrees of freedom are converged within $1 \times 10^{-5} \mathrm{eV}$ and $0.005 \mathrm{eV}^{-1}$, respectively. $6 \times 6 \times 4$ Monkhorst-Pack $k$-point grids[5] and a Gaussian smearing with $0.001 \mathrm{eV}$ half-width are used for Brillouin-zone integrations. Band structures are computed with the DFT $+1 / 2 \operatorname{method}[6]$ as implemented in VASP by Xue et al.[7] In order to explore the relative alignments of FA cations, $\mathrm{FAPbI}_{3}$ and $\mathrm{FAPbBr}_{3}$ systems were computed in a $(2 \times 2 \times 2)$ cell with a $4 \times 4 \times 4 k$ point grid. Structural relaxations for these systems are carried out in internal coordinates. [8] The implementation was improved to retain the initially set symmetry of the crystal.

AIMD runs are carried out with a lower plane-wave cutoff energy of $450 \mathrm{eV}$ and a coarser grid of $2 \times 2 \times 2 k$-points in a $(2 \times 2 \times 2)$ cell. We confirmed that this setup provides band gaps with an accuracy of $0.01 \mathrm{eV}$ as compared to the higher cutoff and finer grid. An NPT ensemble was taken, employing a Langevin thermostat and barostat. [9] Simulations were run at a temperature of $300 \mathrm{~K}$ and a pressure of $0.001 \mathrm{kbar}$ unless noted otherwise. Friction coefficients for each atomic species were set to 5.0. The fictitious masses for the relaxation of the lattice parameters were set to 500. Doubling of this value did not lead to any significant changes in the results for the Cs compounds with I and Br. The fictitious friction coefficients for the lattice parameters were set to 5.0 as well. For the Cs compounds, this led to a destabilization of the structure, as indicated by strongly tilted structures and a significant increase in the deviation of bond-length. Therefore, the friction coefficient for these systems was systematically increased to 100.0 to obtain stable results. Note that this effect is much more pronounced for $\mathrm{Br}$ than for $\mathrm{Cl}$. The reduction of the angle $\beta$ for $\mathrm{CsPbBr}_{3}$ after $7.5 \mathrm{ps}$ indicates that full structural equilibrations for this compound should be carried out with further increased friction coefficients. 

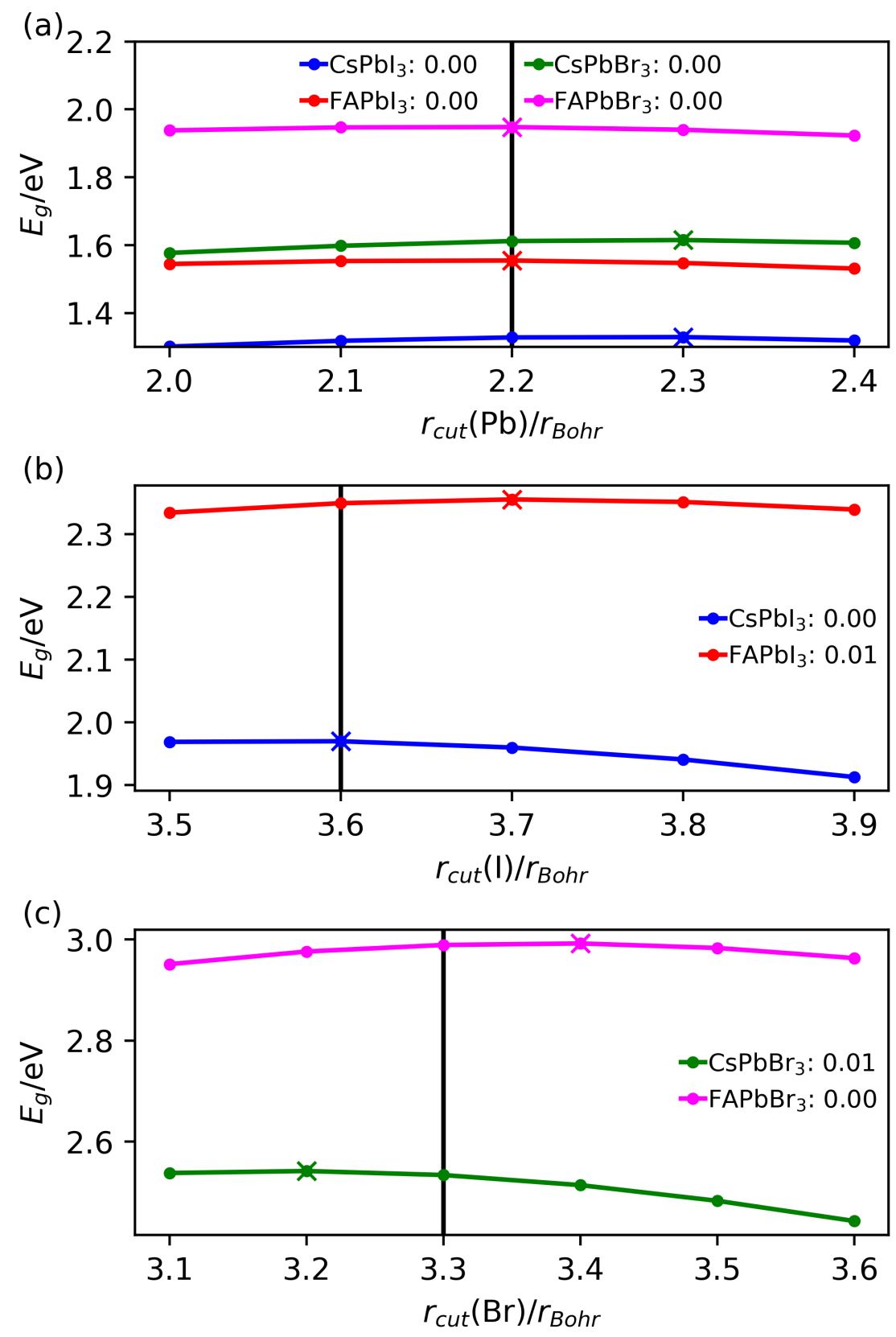

FIG. S9: Optimization of $r_{c u t}(i)$ for $i=\mathrm{Pb}, \mathrm{I}$, and $\mathrm{Br}$. The respective ideal choice (maximum of $E_{g}$ ) is marked by a cross in each data series. The best value for the series of $A B X_{3}$ compounds, obtained from minimizing the collective error to $E_{g}$ is marked as black line in each graph. The resulting error for the $E_{g}$ for each material between ideal and collective $r_{\text {cut }}$ is given in the legend in $\mathrm{eV}$. 
All valence shells contributing to the band edges of the studied compounds have been corrected by the DFT-1/2 method. These are $5 p(\mathrm{I}), 4 p(\mathrm{Br})$, and $6 p(\mathrm{~Pb})$. A-site cations are typically not contributing to the band edges in $A B X_{3}$ halide perovskites, which was confirmed by density of states calculations.

\section{FORMATION ENERGIES}

Formation energies are calculated with respect to the binary phases of the metal-halide compounds. For Cs $X, X=\mathrm{Br}$ and I we compared the $F m \overline{3} m$ and $\mathrm{Pbcm}$ phases. We took these as starting point, replacing $\mathrm{Cs}$ by $\mathrm{FA}$ as starting structures of the FA $X$ compounds. For $\mathrm{Pb} X_{2}$ we chose the $\mathrm{P}_{3} m c$ phase. The thermodynamically favorable phases are summarized in table S1. With these references, formation energies are calculated as

$$
E_{\text {form }}=E_{0}\left(A B X_{3}\right)-E_{0}(A X)-E_{0}\left(B X_{2}\right) .
$$

TABLE S1: Overview of the chosen binary reference phases.

\begin{tabular}{lcccccc}
\hline \hline & $\mathrm{CsI}$ & $\mathrm{CsBr}$ & $\mathrm{FAbI}$ & $\mathrm{FABr}$ & $\mathrm{PbI}_{2}$ & $\mathrm{PbBr}_{2}$ \\
\hline$\left(E_{0} /\right.$ f.u. $) / \mathrm{eV}$ & -8.18 & -8.35 & -47.59 & -48.12 & -9.23 & -10.11 \\
Spacegroup & $F m \overline{3} m$ & $P b c m$ & $P b c m$ & $P b c m$ & $P 6_{3} m c$ & $P 6_{3} m c$ \\
\hline \hline
\end{tabular}

* julian.gebhardt@iwm.fraunhofer.de

[1] G. Kresse and J. Furthmüller, Efficiency of ab-initio total energy calculations for metals and semiconductors using a plane-wave basis set, Comput. Mater. Sci. 6, 15 (1996).

[2] P. E. Blöchl, Projector augmented-wave method, Phys. Rev. B 50, 17953 (1994).

[3] J. P. Perdew, K. Burke, and M. Ernzerhof, Generalized Gradient Approximation Made Simple, Phys. Rev. Lett. 77, 3865 (1996).

[4] A. Tkatchenko and M. Scheffler, Accurate Molecular Van Der Waals Interactions from GroundState Electron Density and Free-Atom Reference Data, Phys. Rev. Lett. 102, 073005 (2009). 
[5] H. J. Monkhorst and J. D. Pack, Special points for Brillouin-zone integrations, Phys. Rev. B 13, 5188 (1976).

[6] L. G. Ferreira, M. Marques, and L. K. Teles, An Approximation to Density Functional Theory for an Accurate Calculation of Band-gaps of Semiconductors, Phys. Rev. B 78, 125116 (2008), arXiv:0808.0729.

[7] K.-H. Xue, J.-H. Yuan, L. R. Fonseca, and X.-S. Miao, Improved LDA-1/2 method for band structure calculations in covalent semiconductors, Comput. Mater. Sci. 153, 493 (2018).

[8] T. Bučko, J. Hafner, and J. G. Angyán, Geometry Optimization of Periodic Systems Using Internal Coordinates, J. Chem. Phys. 122, 124508 (2005).

[9] M. P. Allen and D. J. Tildesley, Computer Simulation of Liquids, 2nd ed. (Oxford University Press, Oxford, 2017). 